\title{
Contribution Du Management Par La Qualité A L'étude Systémique Des Dimensions De La Réforme Administrative: Revue De Littérature Et Proposition D'un Modèle Conceptuel Pour Le Service Public
}

\author{
Abdelmajid Haddad, Doctorant \\ Saïd Mssassi, Professeur \\ Mohamed Makkaoui, Professeur \\ Ecole Nationale de Commerce et de Gestion de Tanger \\ Université Abdelmalek Essaâdi, Morocco
}

Doi:10.19044/esj.2020.v16n13p232 URL:http://dx.doi.org/10.19044/esj.2020.v16n13p232

\section{Resume}

Le présent article a pour objectif d'analyser la contribution du management par la qualité à la réforme administrative. Le choix méthodologique consiste en une synthèse théorique des modèles de la qualité de service ainsi qu'une analyse documentaire et théorique des expériences des Etats en matière de la Réforme. La complexité de celle-ci n'était pas maîtrisée par les modèles de Réforme adoptés depuis un demi-siècle. Toutefois, le modèle du New Public Management a bénéficié largement du management par la qualité et de son approche systémique pour atteindre les résultats escomptés. Ceci a démontré qu'il existe une relation entre le management par la qualité et la maîtrise de la réforme administrative. Ce faisant, par une synthèse théorique et selon une approche systémique, cet article propose un modèle conceptuel permettant de comprendre comment Quatre dimensions qualitatives du service public influencent la satisfaction du citoyen-client. Cette satisfaction aide à harmoniser la relation entre les parties prenantes de la Réforme à savoir: les managers publics, les hommes politiques et les citoyens-clients. Grâce à cette harmonisation, le management par la qualité favorise la maitrise de la complexité de la Réforme et conduit ainsi à sa réussite. Ceci est exprimé sous forme de cinq hypothèses qui complètent ce modèle.

Mots-clés: Réforme administrative, Management public, Management par la qualité, Satisfaction, Service public 


\title{
Quality Management Contribution to The Systemic Study of the Administrative Reform Dimensions: Literature Review and Proposal of a Theoretical Model for The Public Service
}

\author{
Abdelmajid Haddad, Doctorant \\ Saïd Mssassi, Professeur \\ Mohamed Makkaoui, Professeur
}

Ecole Nationale de Commerce et de Gestion de Tanger

Université Abdelmalek Essaâdi, Morocco

\begin{abstract}
This paper focuses on analysing the contribution of quality management to the administrative reform. The methodological choice consists of a theoretical synthesis of the models of quality of service as well as a documentary and theoretical analysis of the experiences of the States with regard to the Reform. The complexity of this was not mastered by the Reform models adopted for half a century. However, the New Public Management model has benefited greatly from quality management and its systemic approach to achieve the expected results. This demonstrated that there is a relationship between quality management and mastery of administrative reform. In doing so, through a theoretical synthesis and according to a systemic approach, this article proposes a conceptual model making it possible to understand how Four qualitative dimensions of public service influence the satisfaction of the citizen-client. This satisfaction helps to harmonize the relationship between the stakeholders of the Reform, namely: public managers, politicians and citizen-clients. Thanks to this harmonization, quality management promotes mastery of the complexity of the Reform and thus leads to its success. This is expressed in the form of four hypotheses which complete this model.
\end{abstract}

Keywords: Administrative Reform, Public management, Quality management, Satisfaction, Public service

\section{Introduction}

La réforme administrative est une opération complexe qui implique l'intervention directe de deux acteurs de différentes orientations et de 
préoccupations dissemblables. Au premier niveau, les hommes politiques sont les responsables de l'élaboration du programme de la réforme et de choix de son modèle approprié. Au second rang, les managers publics sont en charge de mettre en œuvre ce programme de la réforme selon le modèle choisi. Les premiers s'intéressent aux résultats de la réforme tandis que les deuxièmes se préoccupent des moyens de mise en œuvre. L'incompatibilité des objectifs politiques de la réforme avec les contraintes du management de l'administration publique produit des tensions entre ces deux acteurs et influe directement sur la qualité du service public. Cette conséquence est subie par un troisième acteur de la réforme, aussi important que les premiers, qui change de statut et de position. Il s'agit de l'usager du service public, devenu Citoyenclient. Sa satisfaction évolue vite et devient ainsi un élément de pression sur les deux premiers acteurs de la réforme.

En plus, la rationalité juridique qui anime les concepteurs de la réforme administrative ne laisse pas assez de manœuvres à la rationalité managériale. Ceci complique l'échange entre les trois acteurs et principales parties prenantes de la réforme administrative : les hommes politiques (concepteurs de la réforme), les managers publics (exécuteurs de la réforme) et les citoyensclients (à la fois demandeurs et bénéficiaires de la réforme). La réforme devient ainsi une action complexe. Faute d'approches utilisées pour maîtriser cette complexité, les modèles de la réforme mobilisés jusqu'à présent n'ont pas pu maîtriser la relation entre ces parties prenantes.

Dans ce papier, les auteurs proposent un nouveau modèle de réforme alternatif capable de traiter cette complexité et de maîtriser les relations entre les hommes politiques, les managers publics et les citoyens-clients. Selon une approche systémique, ce modèle est conçu principalement pour introduire un management par la qualité du service public dans l'administration publique. En effet, la qualité du service public influe sur la satisfaction des citoyensclients et réduit ainsi les pressions qu'ils exercent sur les hommes politiques et les managers publics. Ceci permet encore de faire disparaitre les tensions qui existent entre les managers publics et les hommes politiques lors de la mise en œuvre de la Réforme administrative.

Ce modèle est fondé sur un système de quatre dimensions qualitatives du service public. Il nous aide à comprendre dans quelle mesure le management par la qualité est susceptible de contribuer à la gestion de la crise en modèles de Réforme dont souffre le service public. Ce type de management permet de résoudre une problématique à l'origine de nature politico-juridique. En effet, ce travail met également l'accent sur l'apport du management à la résolution des problèmes publics souvent affectés à des disciplines dominantes dans ce secteur.

Sous forme d'une synthèse théorique, cet article présente, en premier lieu, une analyse documentaire des expériences de la réforme administrative, 
au niveau mondial. Il s'agit d'une synthèse des différents modèles de réforme du management public appliqués depuis plus d'un demi-siècle (1960-2010). Ensuite, une synthèse théorique des modèles de la qualité de service sera élaborée également. Ces deux synthèses théoriques, issues également des études empiriques précédentes, ont favorisé «une compréhension interprétativiste » (Melhaoui \& Belhadj, 2020) du rôle du management par la qualité dans la maîtrise de la Réforme administrative. Ce rôle sera présenté sous forme d'influence de quatre dimensions qualitatives du service public sur la satisfaction et la confiance des citoyens-clients ainsi que sur la maîtrise des pressions et tensions caractérisant la relation entre les trois parties prenantes de la Réforme. Finalement, ces relations seront présentées par un modèle conceptuel issu de la revue de littérature.

\section{Crise de modèle de réforme administrative}

La réforme de l'administration publique et de son management a subi plusieurs vagues de changement. Ces réformes ont conduit à des modifications insatisfaisantes au niveau du système administratif mondial. Ce sont les parties prenantes de cette réforme qui ne sont pas satisfaites malgré leur rôle principal. Les premières tentatives de réforme ont pris des formes développées dans le cadre du modèle Wébérien qui présente le premier modèle de mangement de l'administration publique. Ce modèle fondé sur les principes de la bureaucratie n'a pas duré longtemps pour devenir critiqué et cause principale de tous les maux dont souffre l'administration publique.

$\mathrm{Vu}$ ces critiques grandissantes du modèle Wébérien, les chercheurs et les concepteurs ont opté pour un nouveau modèle inspiré des méthodes et pratiques ayant fait preuve des résultats positifs dans le secteur privé. Ces méthodes de management de l'entreprise privée présentent les nouvelles révélations du nouveau modèle de réforme, le New Public Management. Ce dernier n'a pas résisté longtemps pour devenir lui aussi la cible des critiques issues de l'insatisfaction des parties prenantes de l'action publique.

Pour faire face à ces critiques, les théoriciens [(Hood, 1991); (Osborne \& Gaebler, 1992) ; (Moore, 1995) ; (Bouckaert, 2003) ; (Osborne, 2006) ; (Politt \& Bouckaert, 2011); (Torfing \& Triantafillou, 2013)] ont cherché d'autres modèles qui peuvent réaliser la satisfaction des acteurs du management public. Ceci a favorisé la conception de nouveaux modèles qualifiés de Post NPM (New Public Management). Ces derniers ont intégré également la vague des critiques des modèles de la réforme.

Dans la plupart des cas, ils ont été jugés comme des modèles hybrides issus des deux précédents modèles : le modèle Wébérien et le NPM. Ce statut quo de la réforme montre clairement qu'il y a une crise de modèle de changement capable de gagner la confiance et la satisfaction des parties 
prenantes de l'action publique à savoir : les hommes politiques, les mangers publics et les citoyens-clients.

Le tableau $\mathrm{n}^{\circ} 1$ (ci-dessous) suivant présente les principales limites et critiques qui ont accompagné les différents modèles de réforme de l'administration publique: 


\begin{tabular}{|c|c|c|}
\hline Modèle de réforme & Limites & Auteur \\
\hline \multirow{6}{*}{$\begin{array}{l}\text { La Bureaucratie ou } \\
\text { Modèle Wébérien } \\
\text { Les années 1960-1970 }\end{array}$} & -Implication limitée des citoyens. & Johnson, D (2006) \\
\hline & -Préservation des avantages des membres de l'administration au détriment des intérêts des clients. & \multirow[t]{2}{*}{ Dupuy, F (2000) } \\
\hline & $\begin{array}{l}\text {-Influence négative des fonctionnaires sur la formulation et l'implantation des politiques publiques et sur } \\
\text { la démocratie. }\end{array}$ & \\
\hline & $\begin{array}{l}\text {-Modèle fondé sur la notion d'intérêt public assuré par le contrôle politico-démocratique et l'imputabilité } \\
\text { de l'administration. } \\
\text {-Il implique une distinction bien établie entre organisations publiques et entreprises privées. }\end{array}$ & Perry \& Rainey (1988) \\
\hline & $\begin{array}{l}\text {-Les fonctionnaires sont des « bureaucrates » et veillent davantage à administrer des lois qu'à offrir des } \\
\text { services publics en vue d'atteindre des résultats précis. } \\
\text {-Faible capacité à répondre aux demandes sociétales changeantes. } \\
\text {-Le travail des cadres administratifs est limité au respect des règles et procédures. }\end{array}$ & Aucoin, P. (1995) \\
\hline & Modèle inefficace dans un contexte d'action dynamique et complexe. & Michaud, N. (2019) \\
\hline \multirow{8}{*}{$\begin{array}{l}\text { Le New Public Management } \\
\text { Les années 1980-1990 }\end{array}$} & $\begin{array}{l}\text {-Il n'existe aucune évidence empirique de l'amélioration des résultats des administrations, résultats } \\
\text { immédiats (outputs), effets (Outcomes) ou processus. }\end{array}$ & Trosa, S. (2000) \\
\hline & $\begin{array}{l}\text { L'incompatibilité des spécificités des deux secteurs public et privé remet en question la transposition des } \\
\text { principes managériaux et de méthodes de gestion de l'entreprise privée dans l'administration publique }\end{array}$ & $\begin{array}{l}\text { Chevalier, G. (2009) ; Allison, G. (1980) ; Henry, N. } \\
\text { (1999); Bellé \& Ongaro (2014) }\end{array}$ \\
\hline & -La rationalité juridique du secteur public est différente de la rationalité managériale du secteur privé. & Chevalier, J. et al. (1982) \\
\hline & $\begin{array}{l}\text {-L'administration est influencée par le droit administratif notamment le fameux statut de la fonction } \\
\text { publique et la conception même de l'État en tant qu'élément moteur de la société. }\end{array}$ & Gualmini et al. (2006) ; Ongaro E. $(2008,2010$ et 2012) \\
\hline & $\begin{array}{l}\text {-Différence entre le statut du client de l'entreprise et celui de l'usager du service public. } \\
\text {-Confusion entre « individu - citoyen - consommateur - client ». }\end{array}$ & $\begin{array}{l}\text { Pesqueux, Y. (2006); } \\
\text { Jeannot, G. (1998) }\end{array}$ \\
\hline & $\begin{array}{l}\text {-Modèle de réforme imposé par les dirigeants politiques qui mettent la pression sur les fonctionnaires. } \\
\text { - Modèle qui fait partie du Marketing politique. }\end{array}$ & $\begin{array}{l}\text { Pesqueux, Y (2006) } \\
\text { Mazouz, B. et al. (2015) }\end{array}$ \\
\hline & -Objectifs contradictoires du NPM. & Politt \& Bouckaert (2011) \\
\hline & -Nouveau-né de l'économie néoclassique et en particulier de la théorie du choix public. & Osborne, S. P. (2006) \\
\hline $\begin{array}{l}\text { La Valeur Publique } \\
\quad(1990-2000)\end{array}$ & \multirow[t]{4}{*}{$\begin{array}{l}\text {-Modèles hybrides (NPM, bureaucratie) caractérisés par les mélanges, la complexité et la stratification. } \\
\text {-Modèle plaçant distinction entre Etat et marché. }\end{array}$} & \multirow{4}{*}{$\begin{array}{l}\text { Osborne, S. P. (2006); Politt \& Bouckaert (2011); Light, } \\
\text { P. (1997) ; Talbot \& Johnson (2007) ; Christensen et al. } \\
\text { (2007); Streck \& Thelen (2005) ; Lampropoulou, M., \& } \\
\text { Oikonomo, G. (2018) }\end{array}$} \\
\hline $\begin{array}{l}\text { Le Nouveau Service Public } \\
\quad(2000-2005)\end{array}$ & & \\
\hline $\begin{array}{l}\text { La Nouvelle Gouvernance } \\
\text { Publique (2006-2009) }\end{array}$ & & \\
\hline $\begin{array}{l}\text { Le Nouvel État wébérien } \\
(2010)\end{array}$ & & \\
\hline
\end{tabular}

Tableau n $^{\circ}$ 1: Les limites des modèles de réforme du management public de 1960 à 2010 


\section{Complexité de la réforme et apport du management par la qualité 2.1. La contribution de l'approche systémique à la maîtrise de la complexité de la réforme}

Afin de contribuer à sortir de cette crise de modèle, ce papier tente de démontrer que la complexité de la réforme n'a pas été traitée, dans le cadre des précédents modèles, selon l'approche adéquate. Dans le cursus académique des cadres de l'administration publique, nous remarquons une concentration sur l'approche cartésienne qui ne traite les problèmes publics complexes que d'une manière séparée les uns des autres. Or, Un problème complexe affecte en général un système complexe (Chevalier, 2009) qui gère des relations complexes entre l'administration publique, ses usagers et son environnement (Delorme, 1999). En plus, les deux notions «complexité » et « système » - accompagnant la réforme - sollicitent les acteurs publics à penser à un renouveau managérial que le secteur public en a besoin d'urgence [(Chevalier, 2009) ; (Morin, 2013)]. La complexité du système administratif s'aggrave du fait qu'il est entouré par des systèmes technologiques, politiques et économiques qui sont eux aussi complexes (Homer-Dixon, 2002).

En général, dans le cadre des réformes précédentes, les organisations publiques composées d'un système de plusieurs entités multifonctionnelles complexes tentent d'accorder des objectifs et des contraintes contradictoire [(Perrow, 1972);(T. Christensen et al., (2012)]. Le service public est par nature un service complexe (ERMES, 1988). Il est évolutif dans le temps et dans l'espace et transformatif historiquement et socialement (Bauby, 2016). C'est pourquoi, il n'en existe pas un service public unique et universel mais un service public complexe. D'ailleurs, les réformes d'adaptation du service public interviennent souvent par cycles et par vagues (Modèle Wébérien, NPM, NGP, NPS, NEW...) caractérisés par les mélanges et la complexité [(Light, 1997) ; (Talbot \& Johnson, 2007) ; (Christensen et al., 2007) ; (Streck \& Thele, 2005)]. Les réformes administratives sont donc des réformes complexes qui doivent satisfaire les intérêts des structures sociétales, publiques et culturelles de plus en plus complexes (Pollitt \& Bouckaert, 2011). En bref, ce sont des réformes complexes dans des mélanges complexes (Egeberg \& Trondal, 2009).

Pour agir sur cette complexité de la réforme, l'approche systémique est l'approche la plus adéquate [(Streck \& Thelen, 2005) ; (Chevalier, 2009)], car elle donne à l'administration publique les moyens pour agir autrement au sein de son environnement interne et externe. Cette approche permet d'impliquer tous les acteurs publics qui interagissent au sein du système d'interactions et de relations qui existent entre l'administration publique et les différentes parties prenantes de la Réforme. Pour G. Chevalier (2009), l'approche systémique appliquée à la réforme administrative consiste à envisager un 
organisme public qui entretient des relations en interne et avec son environnement dont les limites sont bien définies.

\subsection{La contribution de l'approche systémique du management par la qualité à la maîtrise de la complexité de la réforme administrative}

Le modèle de réforme recherché doit donc tenir compte de l'ensemble des éléments de ce système complexe ainsi que de son environnement. Pourtant, ceci ne peut être assuré que par l'adoption de l'approche systémique qui semble inexistante lors la de conception des modèles de réforme précédents. En effet, le modèle de réforme du management public par la qualité de service que nous proposons est considéré comme une approche essentiellement systémique (Spencer, 1994). D’ailleurs, à l'origine, selon W.E. Deming (1993), le management par la qualité est un système d'apprentissage approfondi (System of profond knowledge) fondé sur une approche systémique de l'organisation publique. G. Chevalier (2009) précise que la vision systémique de ce Mangement a pour but d'améliorer la qualité finale du service public. Dans ce système, les Inputs ne sont que des entrées par la finalité de l'organisme public, par ses métiers et par les besoins et exigences de ses parties prenantes. Quant à L. Budd (2007), le management par la qualité conduit à une perspective holistique du système politicoadministratif prenant en considération -à notre sens- la centralité du citoyen et de sa satisfaction mais aussi de sa confiance dans les services publics.

En effet, le management par la qualité est adapté aux spécificités publiques par rapport au mouvement dominant du managérialisme (Chanlat, 2003) et correspond plus particulièrement au secteur public qu'au secteur privé (Cannac, 2004). De surcroît, ce type de management comporte à la fois une large base théorique et méthodologique (Gogue, 1997) et une base empirique particulièrement riche d'enseignements [(Singh et al., 2006); (Fryer et al., 2007)]. Il s'agit d'une méthode de management systémique global qui concerne aujourd'hui pratiquement tous les aspects du management d'un organisme public (Chevalier, 2009). Ce faisant, le modèle de management par la qualité devient un nouveau paradigme de management (Gogue, 1997) indépendamment des autres modèles qui ont bénéficié de ses atouts notamment le New Public Management (Peters \& Waterman, 1983).

Dans le cadre des réformes citées précédemment (Light, 1997), le modèle NMP a adopté les pratiques de gestion de la qualité de service depuis les années 1980 et partout dans le monde. Le service rendu à la clientèle, ou la conscience de la qualité, est devenu une stratégie du Mangement Public. Elle porte essentiellement sur la satisfaction des attentes des (citoyens) clients et sur la qualité de service (d'office public) (Wagenheim \& Reurink, 1991).

La réforme du management par l'amélioration de la qualité du service public est située, selon Politt \& Bouckaert (2011), dans un système politique, 
une culture de la société civile, un système de rapports entre politiciens et hauts fonctionnaires, des normes et symboles organisationnels issus de l'histoire et des aspirations du monde économique qui, elles aussi, peuvent varier par pays.

En réalité, la vague de réforme administrative a largement bénéficié des outils et méthodes du management par la qualité. En effet, le Total Quality Management (TQM) a été adopté pour développer des systèmes de qualité dans l'administration publique dans le monde entier (Karasoy, 2018). Selon A. Hatchuel (1998), le mouvement du mangement par la qualité a introduit dans le secteur public, au début des années 90, une vague de rationalisation de l'action publique. La première expérience a été vécue aux États-Unis, au début des années 1980, par le Maire de la ville MADISON ${ }^{11}$ après sa participation à une conférence de Deming sur le management de la qualité. Ce dernier a appliqué ce mangement pour réduire les coûts et améliorer le fonctionnement $\mathrm{du}$ garage municipal. Les résultats satisfaisants de cette application ont encouragé d'autres administrations en dehors même des Etats Unies à pratiquer le management par la qualité. L'évolution du management public par la qualité s'est développée également avec la publication des chartes de service public qui exigent l'amélioration de la qualité du service public afin de satisfaire les attentes des citoyens considérés dorénavant comme citoyenclient. Parmi ces chartes nous citons :

- Les chartes de Singapour et de la Malaisie publiées dans les années 1990

- $\quad$ « Citizen’s Charter » (Charte citoyenne) lancée par le Royaume-Uni en 1991

- La Charte des Services Publics, publiée en France, en 1992.

- La Charte des utilisateurs des Services Publics, adoptée en Belgique en 1992.

- la Carta dei servizi (Charte des services) lancée en Italie en 1993.

- L'initiative «Putting Customers First » publiée aux Etats Unis en 1993.

- L'Initiative des normes de service adoptée au Canada en 1994.

- Le programme « Serving the Community » lancé à Hong Kong en 1995.

- Le programme «Putting Service First » lancé en Australie, au niveau fédéral en 1997.

- La Charte de service public «Public Service Charter » lancée en Corée du Sud en 1998.

- La loi sur le service aux citoyens approuvée par le Parlement en Suède en 1998 (Torres, L. 2005).

\footnotetext{
${ }^{11}$ La ville de Madison est la capitale de l'État du Wisconsin aux États-Unis
} 
Les chartes citées ci-dessus ont favorisé l'introduction des normes de la qualité dans le management des administrations publiques. Elles sont appelées aussi « chartes de service public » ou «chartes de citoyen». Ces chartes ont introduit la culture de mesure de satisfaction des citoyens-clients. Ceci a rendu les réformes au sein de l'administration de plus en plus axées sur le citoyen et sa satisfaction. Selon G. Anne (2010), l'administration a commencé à utiliser le terme «client» au lieu d'usager grâce à la nouvelle conception des bénéficiaires du service public introduite par les chartes de service public.

Le recours aux chartes reflète le souci de l'administration à améliorer la qualité des services publics dans une perspective de satisfaction de ses clients. Ceci a influencé les réflexions sur la réforme au sein des Etats au travers le monde.

En effet, la plupart de ces Chartes recommandent la mise en place d'un dispositif d'évaluation de la qualité du service public et de mesure de la satisfaction des citoyens-clients. Elles mettent en évidence un des principes de la qualité du service public (Cluzel-Métayer, 2006) qui exige: l'accessibilité du service et l'amélioration de la prestation. La première condition concerne l'égalité d'accès au service. Elle met l'accent sur l'adaptation de l'offre de service aux différentes situations sociales et géographiques des citoyensclients. Il s'agit aussi de mettre les informations à la disposition des clients et leur simplifier les démarches administratives afin de renforcer la lisibilité de l'action publique. Quant à la deuxième, la fourniture du service public doit être caractérisée par une attention particulière réservée à l'écoute des clients et à la prévention du contentieux avec lui. Il s'agit aussi de renforcer la proximité, d'accélérer la vitesse de la procédure administrative et d'éviter le retard de délivrance.

Ainsi, ces chartes ne sont qu'une forme des démarches d'amélioration de la qualité, du service public, introduites dans les réformes administratives. Dans son fascicule, L'IEAP (Institut Européen d'administration publique), considère la charte qualité comme une déclaration unilatérale adoptée et publiée par un organisme public qui s'engage à établir et rendre public les normes de mangement de ses services en harmonie avec ses attributions selon la législation et la réglementation en vigueur. C'est une charte de citoyen qui lui permet de s'adresser à son administration aussi directement que possible et qui place la qualité de service attendue au centre de ses promesses.

Le mouvement de la qualité du service public ne s'est pas arrêté à ce stade, les administrations publiques ont commencé a développé leurs propres référentiels de management par la qualité comme le cas du Cadre d'Autoévaluation des Fonctions publiques « $\mathrm{CAF}$ ». Il s'agit d'un référentiel global d'autoévaluation facile à utiliser par les managers publics et qui les aide à évaluer leurs organismes publics. Il traduit la simplification du modèle d'excellence de l'EFQM pour l'adapter aux spécificités du service public 
européen. Sa vocation est d'être un instrument de management dans les organisations publiques européennes (au niveau national/fédéral, régional ou local) qui permet l'amélioration de la performance grâce à la maîtrise des techniques de management par la qualité (Pauliat, 2004). Une autre vocation du CAF, qui nous semble cohérente avec les propos de ce papier concernant l'approche de réforme, est d'être conçu dans le cadre d'une réforme selon une approche systémique (Pauliat, 2004).

\section{Les dimensions de la réforme administrative au sein du système de management par la qualité : Hypothèses et modèle}

Selon plusieurs auteurs (Swiss, 1992), l'administration publique et implicitement le management public semble avoir besoin de développer un modèle de management par la qualité propre au service public. Ce modèle doit prendre en considération la complexité de la réforme qui doit satisfaire les différentes parties prenantes du service public. Ces parties prenantes sont définies par la «stakeholders theory» de R.E. Freeman (1983) qui s'est développée en management stratégique. Cette théorie s'applique au management public et à l'analyse des politiques publiques [(Fouchet, 1999); (R. McAdam et al., 2005)]. Dans le contexte du service public, trois parties prenantes sont identifiées: les managers publics, les hommes politiques (Ministres et députés issues des partis politiques) et les citoyens-clients.

Dans ce sens et selon une approche systémique, ce papier propose un modèle de réforme du management public selon une approche systémique et quadri-dimensionnelle de la qualité du service public. Ce modèle prend en considération la dimension politique de la réforme et le nouveau statut de l'usager du service public. Ce modèle permet d'évaluer la qualité du service public, de mesurer le degré de satisfaction du Citoyen-client et de maîtriser les relations entre la politique publique et la prise de décision du management public.

\subsection{Le système quadridimensionnel de la qualité du service public et la maîtrise de la satisfaction du citoyen-client: Relation au cour de la réforme administrative}

Le caractère quadridimensionnel de notre approche tire ses origines du caractère multidimensionnel du concept de la qualité de service (Fey et Gogue, 1991). On y trouve des chercheurs qui limitent les dimensions qualitatives du service à deux [(Grönroos, 1982) ; (U. Lehtinen et al., 1982) ; (G. Mels et al., 1997)] et d'autres qui identifient trois dimensions (Rust, R.T. et al. , 1994), ou cinq dimensions (A. Parasuraman et al., 1985), voire même dix dimensions (A. Parasuraman et al., 1985). Le présent article identifie quatre dimensions qualitatives à savoir : la dimension institutionnelle, la dimension interactive, la dimension physique et la dimension environnementale ou de Réseau. Ces 
dimensions constituent le cœur du système de management par la qualité du service public.

\subsubsection{La dimension Institutionnelle et satisfaction}

Selon Eiglier et Langeard (1987), le modèle de qualité de service requiert une dimension «qualité d'organisation interne ». Dans leur système de servuction, ces derniers divisent la qualité institutionnelle en deux parties, une partie visible et l'autre non visible par le citoyen-client. La qualité de la partie visible concerne la qualité du support physique et du personnel en contact. Cette qualité est conditionnée par la partie non visible «le système d'organisation interne ». Les auteurs soulignent que ce système est déterminé par les objectifs fixés par l'organisation de service, par sa structure et par les opérations qu'elle réalise. Ce dernier regroupe toutes les fonctions classiques et spécifiques de l'administration (ressources humaines, services juridiques, finance, marketing, système d'information, etc.), nécessaires à la réalisation du service public. Nous pouvons regrouper ces éléments et les associer à leur mot spécifique : le management de l'organisation.

De leurs parts, Lehtinen et Laitamaki (1984) estiment que l'image qu'a construite le citoyen-client du prestataire du service public est une qualité visible qui constitue le résultat du système d'organisation interne. En effet, le citoyen-client collecte des informations sur l'administration publique afin de s'en servir dans son processus de prise de décision. Ces informations véhiculées l'aident à choisir le comportement à adopter lors de sa participation à la réalisation-consommation du service public. Nous pouvons citer par exemple: «si l'image véhiculée de l'administration concernée est corrompue, le citoyen-client peut choisir entre se comporter de la même façon ou de prendre des mesures offensives et dans les deux cas il ne sera pas satisfait du service public ». Cette image de l'administration est influencée aussi par sa politique de communication, le degré de son ouverture sur le citoyen-client, les pratiques de bouche-à-oreille, sa tradition et son idéologie.

Dans le cadre de la réforme du management public, et comme le montrent plusieurs études récentes (R. Belaïd et al., 2009), la qualité institutionnelle de l'administration publique est un facteur explicatif du développement socio-économique. Ce développement est souhaité par l'ensemble des citoyens-clients. C'est un déterminant de leur satisfaction à l'égard des politiques publiques et du rendement de l'administration publique. Il existe aussi des études empiriques qui ont montré l'existence d'une relation positive entre les qualités institutionnelles et la performance des politiques de réforme du management public.

Donc, pour réussir la réforme du management public, l'administration publique doit améliorer d'une manière continue son management interne et veiller à circuler une bonne image du service public. La qualité du 
management interne contribue à produire un service public de qualité qui suscite automatiquement la satisfaction du citoyen-client. Quant à l'image médiatisée de l'administration qui participe à la construction des attentes du citoyen-client, elle doit correspondre à la réalité du service public et être en harmonie avec les perceptions de ses consommateurs. Dans le cadre des services inter-organisationnels, S. Mssassi (2003) considère la dimension institutionnelle comme un déterminant crucial de l'amélioration de la qualité du service (à l'origine public) car le citoyen-client a déjà des attentes par rapport à l'organisation interne du prestataire et de son image.

\subsubsection{La dimension environnementale et satisfaction}

Pache et Paraponaris (1993) estiment que l'administration, en tant que prestataire de service, est appelée à travailler avec des acteurs extérieurs en nouant avec eux des accords de coordination. En d'autres termes, constituer son propre réseau de partenaires qui l'assiste dans le renforcement de ses capacités et la maîtrise de ses incapacités. Selon J.M. Arnaud (1989), Cet environnement de l'administration est impliqué indirectement à différents niveaux dans sa relation avec le citoyen-client. Par ailleurs, S. Mssassi (2003) met cette dimension au même rang d'importance que la dimension institutionnelle dont l'une affecte la qualité de l'autre. C'est pourquoi J.M. Arnaud (1989) considère que le dysfonctionnement de ce réseau peut créer des répercussions négatives sur la prestation totale du service public. Ce qui va affecter la relation avec le citoyen-client notamment sa satisfaction nécessaire au maintien de l'équilibre entre management public et système politique.

En plus, la création d'un environnement de partenaires performants permet à l'administration de s'inscrire dans une logique d'apprentissage mutuel, de gestion des connaissances, de partage des expériences, de disponibilité des compétences professionnelles et de polyvalence des postes (Christophe \& Alain, 2015). Dans cet environnement où règnent un climat de confiance et des modèles d'organisation transversaux avec moins de bureaucratie notamment avec la participation accrue des citoyens-clients au débat public via les réseaux sociaux (Christophe \& Alain, 2015). Dans ce sens, pour structurer et animer son réseau, l'administration doit développer des capacités relationnelles (un partenariat ciblé), contractuelles (des contrats orientés qualité et satisfaction citoyen-client) et organisationnelles (mode de gestion des partenariats). Selon Verschueren \& Wery (2002), ceci nécessite la mise en place d'un réseau qualité professionnel et permanent, responsable de la politique de réseautage et de gestion des partenariats.

\subsubsection{La dimension Physique et satisfaction}

Cette dimension paraît un peu paradoxale à cause de l'intangibilité du service et de son caractère à l'origine non physique. En effet, cette 
caractéristique du service présente un obstacle (Mssasi, 2003) devant le mangement par la qualité du service public. D’ailleurs, Schneider \& Bowen (1992) le confirment et constatent que l'intangibilité du service est la conséquence d'une expérience résultante d'une transaction et moins d'une utilisation d'un bien tangible. Elle rend difficile de s'assurer de la qualité du service avant sa consommation.

Cependant, selon l'approche « produit » de la qualité, le service est considéré comme un produit avec un caractère intangible et immatériel (Levitt, 1976). Cette considération permet de mesurer la qualité du service et facilite ainsi le management par la qualité en dépit des conséquences de l'intangibilité du service public.

Pour J.P. Flipo (1989), l'environnement immédiat du contact avec le client (citoyen) traduit bien cette dimension physique du service public. Il s'agit de l'espace matériel dans lequel se déroule la servuction. Il est composé d'éléments matériels nécessaires à la production du service «public », des équipements et du matériel utilisé par le personnel en contact. L'ensemble de ces éléments constitue le support physique du système de la servuction. Ce support doit être, selon Eiglier \& Langeard (1987), à la fois un outil efficace de communication (la vitrine du service public) et un outil rentable de travail (le système de fabrication du service public). En se basant donc sur des critères liés aux caractéristiques du support physique du service public, l'évaluation de la qualité devienne objective (Boyer \& Nefzi 2009).

Dans ce sens, la qualité du service public dépend aussi de la qualité des éléments tangibles qui accompagnent la production et la consommation du service. L'ensemble de ces éléments peut être présenté sous forme d'indicateurs matérialisant des objectifs de mesure de la qualité physique du service public. Ces indicateurs permettent également de contrôler le degré de satisfaction des citoyens-clients avant même la réalisation du service public. Cette dimension physique du service public est également un déterminant de la satisfaction du citoyen-client aussi important que les deux précédentes dimensions.

\subsubsection{La dimension Interactive et satisfaction}

Selon C. Grönroos (1982), la dimension relationnelle « interactive », traduite par les interactions du personnel en contact avec les clients (aussi citoyens), affecte la qualité du service public. M.R. Solomon et al. (1985) la considèrent comme l'élément indispensable à la réalisation de la servuction nécessaire à l'évaluation de la qualité du service « public » par les « citoyensclients ». N. Nguyen (1991) rajoute que le rôle du citoyen-client doit être pris en compte dans le processus de réalisation du service «public ».

Selon Solomon \& Surprenant (1985), la réussite de la prestation du service « public » est liée fortement à la compétence du personnel impliqué à 
la phase finale de fourniture du service au (citoyen) client. Ce personnel entre en contact direct avec le citoyen-client et devient le prestataire réel du service à ce moment. Son échange avec le citoyen-client et le comportement de l'un et de l'autre accentuent la complexité de la réalisation du service public. Cette interaction de la consommation du service, au moment de sa production, présente, selon l'approche « utilisateur » de la qualité du service, un caractère inséparable du service public. Il s'agit de la simultanéité de la prestation publique et de son usage par le citoyen-client. Cette approche, selon Boyer \& Nefzi (2009), accorde une importance particulière aux facteurs psychologiques, sociologiques et situationnels. Ces derniers interagissent lors de la simultanéité de la production et la consommation du service public et influencent la perception de la qualité du service. Une des définitions contribuant à la maîtrise du concept du service public est que ce dernier soit une expérience temporelle vécue par le citoyen-client lors de l'interaction de celui-ci avec le personnel en contact [Eiglier \& Langeard (1987)] recruté par l'administration publique. Heskett \& Schlesinger (1991) estiment que ce personnel doit être sélectionné en fonction de ses capacités interactives, notamment celles relationnelles. Ces deux auteurs mettent l'accent aussi sur l'importance de la satisfaction et de la formation de ce personnel pour pouvoir améliorer la qualité du service et satisfaire les citoyens-clients.

En effet, vu le nombre important des clients du service public et la pression importante qu'ils peuvent exercer, l'administration publique ne peut pas se permettre d'offrir un service avec une dimension qualitative interactive défaillante (Mssasi, 2003). Leur satisfaction est une condition d'amélioration de la qualité du service public.

La maîtrise de cette dimension se traduit par des actions de renforcement des capacités du personnel en contact à savoir : la motivation et la formation du personnel en contact. La motivation implique aussi la satisfaction du personnel en contact. Cette satisfaction est influencée par des facteurs liés à l'environnement (Fanou \& Giauque, 2018) dans lequel travaille le personnel en contact comme la bonne ambiance au travail, le soutien organisationnel et les conditions générales de travail. La satisfaction du personnel en contact passe aussi par la formation, comme le soulignent $\mathrm{P}$. Bezes et al. (2011). L'amélioration de la qualité du service public appelle à contourner les risques liés au savoir-faire du personnel en contact et son professionnalisme impactant leur autonomie indispensable à la production du service public. À ce niveau, la dimension institutionnelle a un impact significatif sur les qualités interactives.

Plus encore, l'aspect relationnel du métier du personnel en contact transforme le service public en actes socio-politiques, culturels, éthiques et interpersonnels (P. Bezes et al., 2011). Malgré que cette dimension interactive ne soit pas fondamentalement politique, elle a des effets réels sur les politiques 
publiques et les pratiques managériales. La vision managériale de la réforme administrative par la qualité interactive du service public ne vise pas seulement la transformation $\mathrm{du}$ métier $\mathrm{du}$ personnel en contact mais aussi l'administration et les politiques publiques.

-« Hypothèse 1 »: Les quatre dimensions qualitatives du service public (qualité physique, qualité interactive, qualité institutionnelle et qualité environnementale) influenceraient positivement la satisfaction du citoyenclient durant les différentes étapes du processus de sa réalisation ; [(Mssassi, 2003) ; (Solomon \& Surprenant, 1985) ; (Heskett \& Schlesinger, 1991) ; (Lethinen \& Laitamaki, 1984) ; (Eiglier \& Langeard, 1987) ; (Arnaud, 1989)] -« Hypothèse 2 » : Il suffit qu'une dimension qualitative parmi les quatre soit défectueuse pour que la qualité totale du service public soit dégradée aux yeux du citoyen-client. La satisfaction est le résultat du système composé par les quatre dimensions indissociables. [(Mssassi, 2003) ; (Lethinen \& Laitamaki, 1984) ; (Eiglier \& Langeard, 1987)]

\subsection{Les dimensions de la réforme administrative contrôlées par la qualité du service public}

\subsubsection{La relation entre Satisfaction et Confiance des citoyens-clients dans la réforme administrative}

Le rôle de citoyen dans la réussite de la réforme administrative est déterminant du fait qu'il est une partie prenante de l'action publique. Selon M. Amiel (2003), le client du « service public » est également, et par ailleurs, un citoyen. Il transpose inconsciemment et naturellement ses comportements de consommateur de services des organisations privées vers les services publics délivrés par des organisations publiques. En effet, le comportement du citoyen-client influe sur l'aboutissement de la réforme du management public (Vigoda-Gadot, et al., 2018). Son comportement peut ainsi influencer le déroulement de la réforme et ouvrir la voie aux modèles de changement qui l'ont intéressé. Par conséquent, ses choix vont systématiquement impacter l'élaboration des politiques publiques et pousser leur orientation vers sa satisfaction.

Selon plusieurs auteurs, la satisfaction est la seule traduction de la qualité en matière de service (Mssassi, 2003). Force est de constater l'importance de la satisfaction dans la construction d'un système de qualité comme pilier de départ. Eiglier \& Langeard (1987) soulignent que la satisfaction du citoyen-client est une qualité subjective au contraire de la qualité physique du service qui reste objective. Les auteurs considèrent que la dimension importante de la qualité du service public est portée par la qualité subjective : la satisfaction.

Cronin \& Taylor (1992) ont constaté empiriquement la causalité entre la qualité du service et la satisfaction du citoyen-client. En effet, les deux 
auteurs ont conduit 660 questionnaires auprès des clients de quatre services pour montrer que la qualité perçue influence la satisfaction. Ces deux variables ont par conséquent un effet déterminant sur le comportement du citoyen-client et notamment sur sa fidélité et sa confiance. Une fois que l'administration reconnaît le statut du citoyen-client, elle doit procéder à la mesure de sa satisfaction. En effet, plusieurs administrations à l'échelle internationale ont commencé, en plus de la satisfaction du citoyen, à mesurer sa confiance envers les organisations publiques.

Selon Van de Walle \& Bouckaert (2003), les hommes politiques et les journalistes s'inquiètent de plus en plus de la baisse du niveau de cette confiance. Cet état d'esprit des citoyens a conduit à la production des effets néfastes sur l'État et la cohésion de la société. En raison des trois caractériels du service (intangibilité, hétérogénéité et inséparabilité de la production et de la consommation) et contrairement à la qualité et la satisfaction dans le domaine des biens et produits qu'on peut mesurer objectivement par des indicateurs [(Crosby, 1979); (Garvin, 1983); (Van de Walle \& Bouckaert, 2003)], la satisfaction et la qualité des services sont difficiles à mesurer.

Dans ce contexte, il est nécessaire de réconcilier, selon Emery et al. (2011), la rationalité politique (transformée en juridique) et administrative (transformée en managériale) et de travailler les dimensions de la qualité du service public qui sous-tendent la satisfaction des besoins exprimés et implicites des citoyens-clients. Cette satisfaction aide à regagner la confiance des citoyens-clients dans la Réforme. Ces deux auteurs estiment que le management public est soumis à la coexistence de deux statuts des usagers dépendant : le citoyen et le client dont la régulation doit être y afférente.

E. Vigoda-Gadot et al. (2018) appellent les managers publics à s'orienter vers le renforcement de la confiance afin de réduire la pression exercée par les citoyens-clients sur les hommes politiques. Cette pression a pour objet de réformer de nouveau l'administration publique. Parallèlement, Gounaris \& Venetis (2002) soulignent que la littérature académique considère la confiance du client comme une dimension qualitative du service. Ce faisant, pour gérer la relation entre l'administration et les citoyens-clients d'une part et entre l'organisation privée et ses clients d'autre part, la qualité du service est un facteur déterminant pour le renforcement du lien de confiance (Nefzi, 2007).

Il existe donc une causalité entre qualité du service public, satisfaction des citoyens-clients et confiance dans l'administration publique et son modèle de Réforme. En effet, d'après Sirieix \& Dubois (1999) et J. Frisou (2000), la satisfaction est un antécédent de la confiance dans la relation Organisationclient. De surcroît, d'après K.J. Arrow (1972), la confiance fait partie intégrante de toute transaction de consommation effectuée sur une période de temps. L'auteur estime que le retard économique s'explique par le manque de 
confiance mutuelle ou de « croyance mutuelle ». En plus, selon Barney \& Hansen (1994) et F. Fukuyama (1995), la confiance peut être définie comme l'ensemble des attentes exprimées par les membres d'une communauté caractérisée par la domination d'un comportement régulier, honnête et coopératif. Ainsi, sur la base de la confiance, les caractéristiques de la société sont au cœur de la compréhension du succès et de l'échec économique (Belqasmi \& Debbarh, 2017) et par conséquent de la politique de réforme.

Enfin, pour mesurer la satisfaction et la confiance des citoyens-clients, il faut tout d'abord procéder à l'évaluation de la qualité totale du service public. Ainsi, la confiance dans la réforme est influencée par la qualité du service public sous la forme totale de ses quatre dimensions : interactive, physique, institutionnelle et environnementale.

- Hypothèse 3» : Plus la satisfaction est assurée par la qualité totale du service public plus le citoyen-client est confiant dans la Réforme. [(E. Vigoda-Gadot et al., 2018) ; (Mssassi, 2003) ; (Gounaris \& Venetis, 2002) ; (Nefzi, 2007) ; (Sirieix \& Dubois, 1999) ; (Frisou, 2000)]

\subsubsection{La relation entre les pressions des citoyens-clients et les tensions entre managers publics et hommes politiques lors de la réforme administrative}

Ce qui fait la différence entre l'administration et l'entreprise privée c'est la divergence des intérêts des parties prenantes. Outre les managers publics et les citoyens-clients, les hommes politiques influencent la qualité finale du service public indirectement via leur politique publique. Ils sont en général des élus qui ont gagné la confiance des électeurs après présentation d'un programme électoral. Dans ce programme, la politique de réforme et d'amélioration du service public est l'une des plus importantes composantes. En fait, dans un contexte de réponse aux exigences d'ordre économique et social, les hommes politiques mobilisent les administrations publiques pour mettre en œuvre leur politique de Réforme. Généralement, les hommes politiques développent les idées de réforme du management public suite à des pressions exercées par les citoyens-clients (Pollitt \& Bouckaert, 2011).

Cette pression est le résultat d'un changement qui a affecté la vie socioéconomique du citoyen-client. Il peut s'agir de scandales politiques, de crises économiques, de catastrophes naturelles, de mauvaise qualité du service public, etc. De ce fait, le citoyen-client participe indirectement à la formulation des politiques de réformes administratives. D'après Pollitt \& Bouckaert (2011), nous constatons que l'administration publique et les partis politiques ne partagent pas la même considération du rôle que peut jouer le citoyen, client du service public. Cette différence conceptuelle est à la base des conflits qui peuvent surgir entre les hommes politiques, élus par les citoyens-clients, et les managers (hauts responsables) de l'administration publique. 
B. Mazouz et al. (2015) estiment que la nature du bien commun et de l'intérêt général, qui justifient les actions de l'administration, provoque des tensions entre managers publics et hommes politiques. Y. Pesqueux (2006) qualifie ces tensions de fractures, entre les élus et les fonctionnaires, qui se construisent lors de chaque adoption d'une nouvelle réforme. Pollitt \& Bouckaert (2011) expliquent cette constatation par la divergence des préoccupations des deux parties. Les hommes politiques s'intéressent particulièrement aux Outcomes - résultats attendus du service public - de la politique publique alors que les managers sont préoccupés par les Inputs, c'està-dire les moyens et procédures à mettre en place pour mettre en œuvre la politique publique et fournir le service public.

Ces deux auteurs ont intégré également, dans leur modèle de réforme du management public, le rapport économie-coût-effectivité. Il importe de rappeler ici les avantages du mangement par la qualité en matière de réduction des coûts de non-qualité du service public. Outre la différence conceptuelle au niveau du statut de l'usager du service public, nous pouvons énoncer que les mangers publics et les hommes politiques partent des Inputs différents. Ceci provoque des tensions entre les deux.

-« Hypothèse 4» Les citoyens-clients sont d'autant moins satisfaits du service public qu'ils exercent une pression sur les hommes politiques. [(Pollitt \& Bouckaert, 2011) ; (E. Vigoda-Gadot et al., 2018)].

- «Hypothèse 5» Les citoyens-clients sont d'autant moins satisfaits du service public qu'ils deviennent source des tensions entre les hommes politiques et les managers publics. [(B. Mazouz et al., 2015) ; (Pesqueux, 2006) ; (Bouckaert, 2011)].

\subsection{Modèle conceptuel de la réforme administrative par la qualité du service public}

Le présent modèle (voir la figure $\mathrm{n}^{\circ} 1$ ) permet de maîtriser la gestion des relations qui compliquent la réforme administrative. Ainsi, celle-ci est orientée vers la satisfaction et la confiance du citoyen-client nécessaires au maintien de l'équilibre relationnel de la réforme administrative. Cet équilibre est recherché au niveau des relations qui regroupent les managers publics, les hommes politiques et les citoyens-clients. En effet, le système de management par la qualité permet d'assurer l'amélioration continue des quatre dimensions qualitatives du service public indispensables pour satisfaire les citoyensclients et renforcer leur confiance envers le modèle de la réforme. Etant donné que les pressions exercées sur les hommes politiques sont à la fois le résultat d'insatisfaction des citoyens-clients et la source des tensions entre les acteurs de la réforme, l'amélioration de la qualité du service public nous semble la solution adéquate. En effet, la mauvaise qualité du service public devient la raison pour laquelle la conception de ce nouveau modèle peut améliorer la 
satisfaction du citoyen-client, regagner sa confiance et assurer le bon déroulement de la réforme.

Figure $\mathbf{n}^{\circ} 1$ : Modèle conceptuel de Réforme administrative par la qualité du service public

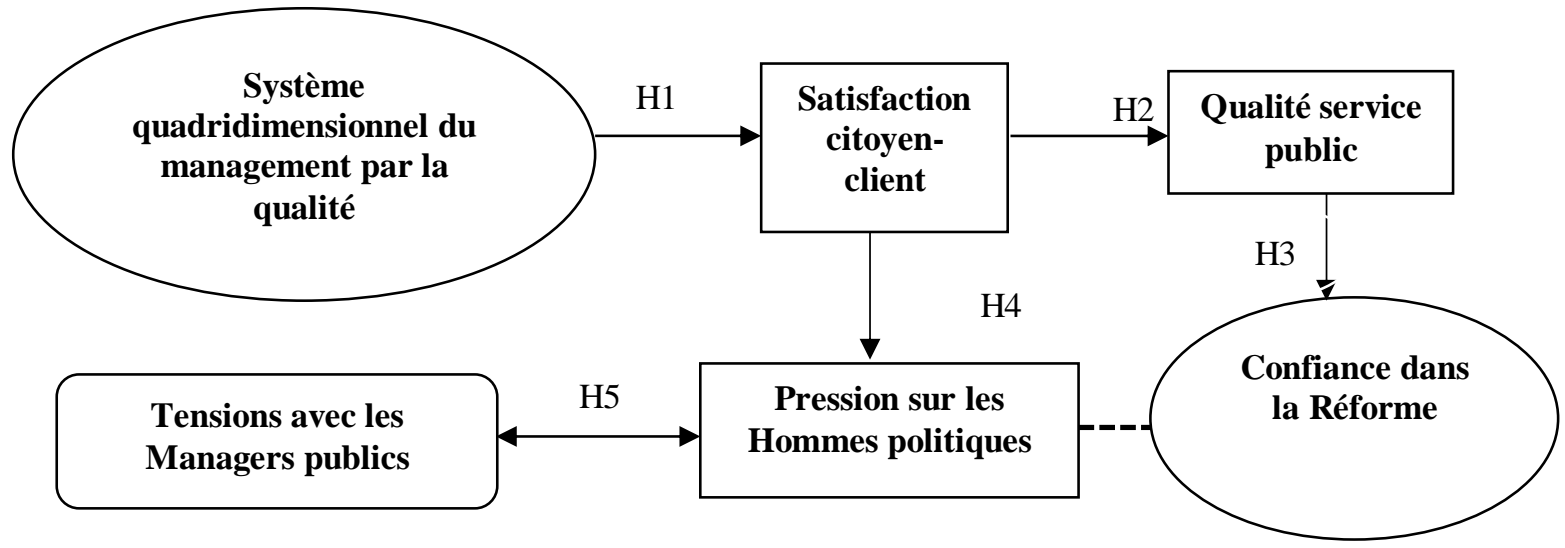

En effet, le bon déroulement de la réforme est condition de la création d'un environnement de confiance qui règne au moment des interactions entre ses parties prenantes : les managers publics, les hommes politiques et les citoyens-clients. Ce modèle permet ainsi de contrôler les relations entre ces parties prenantes de la réforme. Il permet également d'intervenir au moment de pannes au niveau de ces relations à travers l'amélioration continue des dimensions qualitatives du service public indispensables pour atteindre la satisfaction du citoyen-client, axe central de la réforme.

Le fait donc de mettre aussi la qualité du service public au centre de la stratégie de réforme administrative semble adéquat pour atténuer les besoins de nouvelles réformes. Ceci est susceptible de faire éviter la prolifération des modèles insatisfaisants pour ses parties prenantes. Lorsque l'administration publique assure la qualité du service public pour satisfaire les citoyens-clients, le besoin de réformer ne se présente plus car il n'existe plus de pressions ni de tensions qui rendent la relation complexe entre les parties prenantes de la réforme. L'amélioration continue de la qualité du service public par les quatre dimensions conduit efficacement à réduire les pressions en faveur d'une nouvelle réforme.

Enfin, ce modèle quadridimensionnel (de base) de réforme administrative par la qualité du service public, peut être définit comme une méthode (Mssassi, 2003) adoptée par un système administratif qui lui permet d'équilibrer le contenu des interactions au sein du système de la servuction publique par la maîtrise des quatre dimensions qualitatives du service public. Cette méthode permet également d'inspirer le système politique par sa capacité d'amélioration de la satisfaction des citoyens-clients et de renforcement de leur confiance envers les institutions de l'État. D'après ce 
modèle, il existe des liens entre les quatre dimensions de la qualité du service public, Satisfaction et Confiance des citoyens-clients, hommes politiques et managers publics.

\section{Conclusion}

En s'inspirant de l'analyse documentaire et de la revue de littérature, les dimensions qualitatives proposées mettent en harmonie les éléments du système de formulation et de mise en œuvre des réformes administratives. Leur impact sur l'amélioration de la qualité du service public garantie une synergie entre le système politique (les hommes politiques avec leur programme de développement transformé, après les élections, en politiques de Réforme) et l'administration publique chargée de mettre en œuvre ces politiques de Réforme.

Le modèle proposé dans cet article permet, en plus, de combler les lacunes existantes en matière de mesure des réformes administratives. Selon Vigoda-Gadot et al. (2018), la mesure et l'évaluation de la nécessité de nouvelles réformes administratives semblent inexistantes. Ce modèle, fondé sur le management par la qualité, est riche en méthodes et outils de mesure qui aideront à l'évaluation de la réforme et des futures réformes. Dans le cadre du présent modèle, la mesure de la satisfaction, de la confiance et des pressions des citoyens-clients ainsi que les tensions entre managers publics et hommes politiques nous permettent d'évaluer la réforme en cours et la nécessité de conduire de nouvelles réformes.

Toutefois, le modèle présenté dans cet article nécessite une confrontation à la réalité marocaine afin de vérifier la pertinence des aboutissements théoriques. Les prochaines étapes de notre recherche consisteront à étudier empiriquement la validité des cinq hypothèses selon une démarche qualitative par étude de cas. Elles consistent également à étudier la validité de la variable « confiance envers les organisations publiques » dans le cadre d'une étude exploratoire menée auprès des administrations publiques marocaines.

En guise de conclusion, le modèle que propose cet article se présente comme une alternative aux modèles Post New Public Management (NPM) permettant à la fois à l'administration publique et aux partis politiques de s'accorder sur la réforme administrative et conséquemment de satisfaire les citoyens-clients et accroître leur confiance dans la Réforme administrative.

\section{References:}

1. Allison, G. T. (1980). Public and private management: are they fundamentally alike in all unimportant respects?. Cambridge, MA: John F. Kennedy School of Government, Harvard University. pp. 283298. 
2. Amiel, M. (2003). La qualité de services dans les administrations publiques: un défi du changement. Pyramides. Revue du Centre d'études et de recherches en administration publique, (7), pp. 149-164.

3. Anne, G. (2010). La notion de service public. DCB 18 Mémoire d'étude, Enssib, Janvier. p.96.

4. Arnaud, J. M. (1989). Echanges inter-organisationnels de services: nature, rôle et implications des différents intervenants. Revue française du marketing, (121), pp. 67-84.

5. Arrow, K. J. (1972). Gifts and Exchanges. Philosophy \& Public Affairs, Vol. 1, No. 4, pp. 343-362.

6. Aucoin, P. (1995). The New Public Management. Canada in comparative perspective, Montréal, Institut de recherche en politiques publiques. p. 171.

7. Barney, J.B. \& Hansen, M.H. (1994). Trustworthiness as a Source of Competitive Advantage. Strategic Management Journal, Vol. 15, Special Issue: Competitive Organizational Behavior, pp. 175-190.

8. Bauby, P. (2016). Service public, services publics. 2e édition La documentation Française. Paris. p. 174.

9. Belaïd, R., Gasmi, F., \& Virto, L. R. (2009). La qualité des institutions influence-t-elle la performance économique? Le cas des télécommunications dans les pays en voie de développement. Revue d'économie du développement, 17(3), pp.51-81.

10. Belqasmi, S., \& Debbarh, M. A. E. A. (2017, April). Étude de la confiance comme déterminant de l'attractivité des P2I marocaines aux IDE: cas de la P2I de l'industrie automobile à Tanger. 1er colloque international Entreprenariat Management et Croissance,

11. Bellé, N., \& Ongaro, E. (2014). Le NMP, les réformes administratives et la motivation au service public: améliorer le dialogue entre les agendas de recherche. Revue Internationale des Sciences Administratives, 80(2), pp.397-416.

12. Bezes, P., Demazière, D., Bianic, T. L., Paradeise, C., Normand, R., Benamouzig, D., ... \& Evetts, J. (2011). New Public Management et professions dans l'État: au-delà des oppositions, quelles recompositions?. Sociologie du travail, 53(3), pp.293-348.

13. Budd, L. (2007). Post-bureaucracy and reanimating public governance. A discourse and practice of continuity? International Journal of Public Sector Management 20(5): pp.531-547.

14. Cannac, Y. (2004). La qualité des services publics. Commission qualité des services publics. La Documentation française.

15. Chanlat, J. F. (2003). Le Managérialisme et l'éthique du bien commun: la question et la motivation au travail dans les services publics (No. hal-00161554). 
16. Chevalier, G. (2009). Eléments de management public: le management public par la qualité. Afnor. pp.453.

17. Chevallier, J., \& Loschak, D. (1982). Rationalité juridique et rationalité managériale dans l'administration française in Management public. Revue Française d'Administration Publique. anc Bulletin de l'Institut International d'Administration Publique Paris, (24), pp .5394.

18. Christensen, T., Lægreid, P., \& Rovik, K. A. (2007). Organization Theory and the Public Sector. Instrument, Culture and Myth. London : Routledge.

19. Christensen, T., Laegreid, P (2012). Les principes contradictoires de l'organisation des agences : la réorganisation d'une réforme, Revue Internationale des Sciences Administratives 78/4, p. 621-639.

20. Cronin Jr, J. J., \& Taylor, S. A. (1992). Measuring service quality: a reexamination and extension. Journal of Marketing, 56(3), pp.55-68.

21. Crosby, P. B. (1979). Quality is free: The art of making quality certain (Vol. 94). New York: McGraw-hill.

22. Delorme, R. (1999). De l'emprise à l'en-prise. Agir en situation complexe. Entre systémique et complexité, chemin faisant, pp.27-46.

23. Deming, W. E., \& Gogue, J. M. (1988). Qualité: la révolution du management. Economica.

24. Deming, W. E. (1993). A system of profound knowledge.

25. Dupuy, François (2000). Le client et le bureaucrate. DUNOD, 178 p.

26. Egeberg, M. et J. Trondal (2009). National agencies in the European Administrative space: Government Driven, Commission Driven or Networked?. Public Administration 87 (4): pp.779-790.

27. Eiglier P., Langeard E. (1987). Servuction: Le marketing des services. Stratégie et Management, Paris, Mc Graw-Hill. p.159.

28. Emery, Y. \& Fortier, I., (2011). L'ethos public en tant que processus social dynamique. Pyramides. Revue du Centre d'études et de recherches en administration publique, (22), pp.83-114.

29. ERMES (1988). La demande de services complexes des firmes multinationales et l'offre correspondante, étude effectuée pour le commissariat général du plan, Université de Lille I.

30. Fanou, A. V., Giauque, D. (2018). Satisfaction au travail dans les administrations publiques en Afrique : une revue systématique de la littérature. Revue Internationale des Sciences Administratives 84/3, pp. 615-630.

31. Fey, Robert, Gogue Jean-Marie (1991). La maîtrise de la qualité. Paris, Les Editions Economica, p.486.

32. Flipo, J. P. (1989). Marketing des services: un mix d'intangible et de tangible. Revue française du marketing, (121), pp .29-38. 
33. Fouchet, R. (1999). Performance, service public et nouvelles approches managériales. Politiques et management public, 17(2), pp.35-49.

34. Freeman, R. E. (1983). Strategic management: A stakeholder approach. Advances in strategic management, 1(1), pp. 31-60.

35. Frisou, J. (2000). Confiance interpersonnelle et engagement: une réorientation béhavioriste. Recherche et Applications en Marketing (French Edition), 15(1), pp. 63-80.

36. Fryer, K. J., Antony, J., \& Douglas, A. (2007). Critical success factors of continuous improvement in the public sector: a literature review and some key findings. The TQM Magazine, 19(5), pp. 497-517.

37. Fukuyama, F. (1995). Trust: The social virtues and the creation of prosperity (Vol. 99). New York: Free press. p.26,

38. Garvin, D. A. (1983), "Quality on the Line," Harvard Business Review, 61 (September-October), 65-73.

39. Gerhard, M., Christo, B. \& Deon, N. (1997). The dimensions of service quality: The original European Perspective Revisited. Service Industries Journal, 17(1).pp173-89.

40. Gilles, J. (1998). Les usagers du service public. PUF, pp.128, Que saisje ?

41. Gogue, J. M. (1997). Management de la qualité. Paris, Economica.

42. Gounaris, S.P. et Venetis, K. (2002). Trust in industrial service relationships: behavioral consequences, antecedents and the moderating effect of the duration of the relationship. Journal of Services Marketing, 16(7), pp.636-655.

43. Grönroos Christian. An applied service marketing theory. European Journal of Marketing, (7/16), pp.30-41

44. Gualmini, E., \& Capano, G. (2006). La pubblica amministrazione in Italia. Il mulino.

45. Hatchuel, A. (1998). Comment penser l'action collective? Théorie des mythes rationnels. L'action collective, pp.177-202.

46. Henry, N. (1999), Public Administration and Public Affairs, Upper Saddle River (N.J.), Prentice Hall, p.500.

47. Heskett, J. L., \& Schlesinger, L. A. (1991). Breaking the cycle of failure in services. Sloan Management Review, 32(3), pp.17-28.

48. Homer-Dixon, T., \& Montréal, B. (2002). Le défi de l'imagination. Comment résoudre les problèmes de l'avenir, 1.

49. Hood, C. (1991). A public management for all seasons?. Public Administration, vol. 69, $\mathrm{n}^{\circ}$ 1, pp. 3-19.

50. Johnson, D. (2006). Theory of organizational design and management decision making, In: thinking government: public sector management in Canada, 2e éd. Peterborough, Broadview Press, pp.243-314. 
51. Karasoy, H. A. (2018). Quality Management in Turkish Public Management: Challenges and Benefits. European Scientific Journal, 14(5), 11. pp. 11-18.

52. Lampropoulou, M., \& Oikonomo, G. (2018). Modèles théoriques d'administration et schémas de réforme de l'État en Grèce. Revue Internationale des Sciences Administratives, 84(1), pp.111-129.

53. Lehtinen, U., \& Lehtinen, J. R. (1982). Service quality: A study of quality dimensions. Unpublished Working Paper, Helsinki: Service Management Institute, Finland OY.

54. Lehtinen, J. R., \& Laitamaki, J. M. (1984). Applications of service quality and services marketing in private hospitals. Service Management Institute, Finland.

55. Light, P. (1997). The tides of Reform: making government work 19451995. New Haven: Yale University Press.

56. Mazouz, B., Sponem, S., \& Rousseau, A. (2015). Le gestionnaire public en question: La difficile conciliation des logiques bureaucratique et managériale. Revue française de gestion, (5), pp.89104.

57. McAdam R., Hazlett S. A. et Casey C. (2005). Performance management in the UK public sector: addressing multiple stakeholder complexity. International Journal of Public Sector Management, 18(3), pp. 256-273.

58. Melhaoui, I. et Balhadj, S. (2020). Le style pédagogique autonomisant contribue-t-il au développement d'une pré-employabilité des jeunes diplômés: revue de littérature et proposition d'un modèle conceptuel. European Scientific Journal. (4/16). pp.107-130

59. Michaud, N. (2019). Secrets d'États? Les principes qui guident l'administration publique et ses enjeux contemporains. 2e édition. PUQ.

60. Moore, M. H. (1995). Creating public value: Strategic management in government. Harvard university press.

61. Morin, E. (2013). La Méthode-tome 5 L'humanité de l'humanité. l'identité humaine: L'Humanité de l'humanité. L'identité humaine. Le Seuil.

62. Mssassi, S. (2003). Le système de gestion intégrale de la qualité en matière de services industriels: étude des perceptions de la qualité des services bancaires et d'assurance par les entreprises clientes (Doctoral dissertation, ANRT).

63. Nefzi, A. (2007). La relation entre la perception de la qualité et la fidélité : une application à la distribution des parfums et cosmétiques en France, actes du colloque E.Thil.

64. Nefzi, A. et Boyer, A. (2009). La perception de la qualité dans le 
domaine des services : Vers une clarification des concepts. La Revue des Sciences de Gestion. (3). pp. 43-54.

65. Nguyen, N. (1991). Un modèle explicatif de l'évaluation de la qualité d'un service: une étude empirique. Recherche et Applications en Marketing (French Edition), 6(2), pp.83-98.

66. Ongaro, E. (2008) L’organizzazione dello Stato tra autonomia e policy capacity. Soveria Mannelli : Rubbettino.

67. Ongaro, E. (2010). The Napoleonic Administrative Tradition and Public Management Reform in France, Greece, Italy, Portugal, Spain. In: Painter, M. et Peters, B.G. (eds.) Tradition and Public Administration, Basingstoke: Palgrave MacMillan.

68. Ongaro, E. (2012). L'influence de la nouvelle gouvernance Européenne sur la réforme de l'administration publique dans les états de tradition administrative napoléonienne. Plaidoyer pour l'adoption d'un nouvel agenda de recherche", Revue Française d'Administration Publique, (141).pp. 239-46.

69. Osborne, D. et Gaebler, T (1992). Reinventing Government. How the entrepreneurial spirit is transforming the public sector. ADDISONWESLEY PUBL. Co. p.427.

70. Osborne, S. P. (2006). The new public governance? 1. Public Management Review (3/8).

71. Pauliat, H. (Ed.). (2004). La qualité: une exigence pour l'action publique en Europe? Presses Univ. Limoges.

72. Parasuraman A., BerryLeonard L., Zeithaml V. A. (1985). A Conceptual Model of Service Quality and its Implications for Future Research. Journal of Marketing (49) Fall, pp.41-50.

73. Pesqueux, Y. (2006). Le “ nouveau management public" (ou New Public Management).

74. Perrow, C. (1972). Complex Organizations. Glenview : Scott Foreman

75. Pollitt, C., \& Bouckaert, G. (2011). Public management reform: A comparative analysis of NPM, the Neo-Weberian State, and New Public Governance, 3rd edn. Oxford University Press.

76. Pache, G. et Paraponaris, C. (1993), L'entreprise en réseau, Presses Universitaires de France, Collection Que sais-je? Paris.

77. Peters T. et R. Waterman (1983). Le prix de l'excellence. Les secrets de meilleures entreprises, Paris, InterEditions.

78. Rust, R. T. \& Oliver, R. L.(1994). Service quality: Insights and managerial implication from the frontier. Journal of Service Quality, 15(4), 32-43.

79. Schneider B., Bowen D. E. (1992). Personnel / Human Ressources management in the Service Sector » Research in Personnel and Human Ressources Management (10), pp.1-30, Jai“Press. 
80. Singh, P. J., \& Mansour-Nahra, P. (2006). ISO 9000 in the public sector: a successful case from Australia. The TQM Magazine, 18(2), pp.131-142

81. Sirieix 1. \& Dubois p.1. (1999). Vers Un Modèle Qualité Satisfaction Intégrant la Confiance. Recherche et Applications en marketing (3/14). pp: 1-22.

82. Solomon, M. R., Czepiel, J. A., \& Surprenant, C. F. (Eds.). (1985). The service encounter: Managing employee/customer interaction in service businesses. Free Press.

83. Solomon, M. R. \& Surprenant, C. F (1985). The service Encounter, Lexington books.

84. Spencer, B. A. (1994). Models of organization and total quality management: a comparison and critical evaluation. Academy of management review, 19(3). pp. 446-471.

85. Streck, W. \& K. Thelen, eds. (2005). Beyond Continuity. Institutional Change in Advanced Political Economies. Oxford : Oxford University Press

86. Swiss, J. E. 1992. Adapting Total Quality Management to Government. Public Administration Review 52(4).pp. 356-362.

87. Talbot, C. \& C. Johnson (2007). Seasonal cycles in public management: disaggregation and re-aggregation. Public Money \& Management, 27 (1).pp.53-60.

88. Theodore, L. (1976). The industrialization of service. Harvard Business Review, 54(5), pp. 63-74.

89. Torfing J \& Triantafillou P. (2013). What's in a name? Grasping new public governance as a political-administrative system. Paper Presented at the ECPR General Conference, Bordeaux 4-7 September.

90. Torres, L. (2005). Service Charters: Reshaping Trust in Government: The Case of Spain. Public Administration Review 65(6).pp. 687-699.

91. Trosa, Sylvie (2000). Christopher Pollitt et Geert Bouckaert : public management reform, a comparative analysis (compte-rendu). Politiques et management public, vol. 18, $\mathrm{n}^{\circ} 4$, Numéro spécial - "Le management public et la mesure : des lettres aux chiffres" pp. 184-189

92. Van de Walle, S. \& Bouckaert, G. (2003). Comparer les niveaux de confiance des citoyens et de satisfaction des utilisateurs en tant qu'indicateurs de bonne gouvernance. Revue internationale des sciences administratives, 69(3). pp. 363-400.

93. Verschueren, I. \& Wery, P. (2002). Développement de la qualité dans les services publics fédéraux (SPF), Pyramides (5).pp. 53-72.

94. Vigoda-Gadot, E., Cohen, N. \& Tsfati, Y. (2018). Réformer les pays : étude mondiale sur la nécessité de futures réformes managériales dans le secteur public. Revue Internationale des Sciences Administratives 
(4/84).pp. 793-812.

95. Wagenheim, G. D. \& Reurink, J. (1991). Customer Service in Public Administration," Public Administration Review 51(3).pp. 263-270. 